\title{
Langerhans cell histiocytosis in acute leukemias of ambiguous or myeloid lineage in adult patients: support for a possible clonal relationship
}

\author{
Sophia L Yohe ${ }^{1}$, Carrie B Chenault ${ }^{2}$, Emina E Torlakovic ${ }^{3}$, Sheryl L Asplund ${ }^{4}$ and
} Robert W McKenna ${ }^{1}$

${ }^{1}$ Special Hematology Laboratory, Division of Hematopathology, University of Minnesota Medical Center, Fairview, Minneapolis, MN, USA; ${ }^{2}$ ProPath, Dallas, TX, USA $;{ }^{3}$ Laboratory Medicine and Pathobiology, University of Toronto, Toronto, ON, Canada and ${ }^{4}$ Division of Hematopathology, Miraca Life Sciences,

Phoenix, AZ, USA

Four patients presented with acute leukemia of ambiguous or myeloid lineage in association with Langerhans cell histiocytosis and provide evidence suggesting a common origin of the two neoplasms. One patient had a non-constitutional trisomy 21 in both the leukemic blasts and the Langerhans cells indicative of a clonal relationship. A second case expressed CD2, CD13, and CD117 on both the Langerhans cells and the blasts suggesting a possible clonal relationship. All four cases exhibited geographic intermingling of the Langerhans cell histiocytosis and acute leukemia and shared unique features including extramedullary leukemia involving lymph nodes in all cases with Langerhans cell histiocytosis only present in sites involved by acute leukemia. T-cell antigen expression was present in all cases with one meeting criteria for mixed phenotype acute leukemia, T/myeloid, not otherwise specified. These findings support the concept that coexistent Langerhans cell histiocytosis and acute leukemia is clonally related in some cases. Furthermore, these cases of acute myeloid or acute leukemia of ambiguous lineage with Langerhans cell histiocytosis share some unique features suggesting a common underlying neoplastic hematopoietic stem cell.

Modern Pathology (2014) 27, 651-656; doi:10.1038/modpathol.2013.181; published online 1 November 2013

Keywords: acute leukemia; acute myeloid leukemia; ambiguous immunophenotype; clonal; Langerhans cell histiocytosis

Langerhans cells (LCs) are a type of dendritic cell that characteristically express S100, CD1a, and langerin (CD207) and contain ultrastructural Birbeck granules. LC histiocytosis is a neoplastic proliferation of LCs that can present at a single location or at multiple sites. It is a heterogeneous disease that may have a high mortality when multiple sites are involved or may resolve spontaneously as often occurs with single site disease. ${ }^{1}$

Rarely, LC histiocytosis may be associated with various hematologic neoplasms including acute lymphoblastic and myeloid leukemias, other clonal

Correspondence: Dr SL Yohe, MD, Special Hematology Laboratory, Division of Hematopathology, University of Minnesota Medical Center, Fairview,, MMC Box Mayo 609, 420 Delaware Street SE, Minneapolis, MN 55455, USA.

E-mail: yohe0001@umn.edu

Received 1 March 2013; revised 9 August 2013; accepted 20 August 2013; published online 1 November 2013 myeloid neoplasms, and lymphomas. ${ }^{2-4}$ Acute lymphoblastic leukemia is more often associated with LC histiocytosis in children, whereas lymphoma and acute myeloid leukemia are more common in adults, which may reflect the frequency of these diseases in the respective populations. ${ }^{2,4}$ Most cases of acute myeloid leukemia associated with LC histiocytosis appear to be treatment related as they occur years after cytotoxic treatment for LC histiocytosis; however, in some cases, LC histiocytosis occurs concurrently or follows acute myeloid leukemia. ${ }^{2,4}$

The biologic relationship of LC histiocytosis with hematologic neoplasms remains to be fully elucidated. In some instances, LC histiocytosis appears to be incidentally associated without evidence of dissemination or progression, ${ }^{5}$ whereas in others the LC histiocytosis follows an aggressive course. ${ }^{6}$ A clonal relationship between LC histiocytosis and T-lymphoblastic leukemia has been shown in 
Table 1 Patient characteristics

\begin{tabular}{|c|c|c|c|c|c|c|c|c|}
\hline Patient & $\begin{array}{c}\text { Age } \\
\text { (years) }\end{array}$ & $\operatorname{Sex}$ & Diagnosis & Cytogenetics & $\begin{array}{l}\text { Presentation } \\
\text { of } L C H\end{array}$ & $\begin{array}{l}\text { Location } \\
\text { LCH }\end{array}$ & $\begin{array}{l}\text { Location } \\
\text { leukemia }\end{array}$ & BRAFV600E testing \\
\hline 1 & 36 & M & $\begin{array}{l}\text { Acute myeloid leukemia, } \\
\text { NOS, without maturation }\end{array}$ & 46, XY & Concurrent & $\begin{array}{l}\mathrm{LN} \\
\mathrm{BM}\end{array}$ & $\begin{array}{l}\mathrm{LN} \\
\mathrm{BM}\end{array}$ & $\begin{array}{l}\text { Material not } \\
\text { available }\end{array}$ \\
\hline 2 & 54 & M & $\begin{array}{l}\text { AML with myelodysplasia- } \\
\text { related changes }\end{array}$ & Complex $^{b}$ & $\begin{array}{l}\text { Subsequent } \\
\text { ( } 4 \text { months) }\end{array}$ & $\mathrm{LN}$ & $\begin{array}{l}\mathrm{LN} \\
\mathrm{BM}\end{array}$ & $\begin{array}{l}\text { B5 fixed study } \\
\text { failed }\end{array}$ \\
\hline 3 & 80 & $\mathrm{~F}$ & Undifferentiated leukemia & Not performed & Concurrent & LN & $\mathrm{LN}$ & Negative \\
\hline 4 & 68 & M & $\begin{array}{l}\text { Mixed phenotype acute } \\
\text { leukemia, T/myeloid }\end{array}$ & $47, \mathrm{XY},+21$ & Concurrent & $\begin{array}{l}\mathrm{LN} \\
\mathrm{BM}\end{array}$ & $\begin{array}{l}\mathrm{LN} \\
\mathrm{BM}\end{array}$ & Negative \\
\hline
\end{tabular}

Abbreviations: AML: acute myeloid leukemia; BM: bone marrow; LCH: Langerhans cell histiocytosis; LN: lymph node; NA: not applicable; NOS: not otherwise specified.

This case from 1999 was originally diagnosed as biphenotypic leukemia by the EGIL criteria, ${ }^{11}$ but would be classified as acute myeloid leukemia, NOS, without maturation by the current WHO 2008 Classification system.

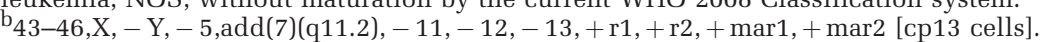

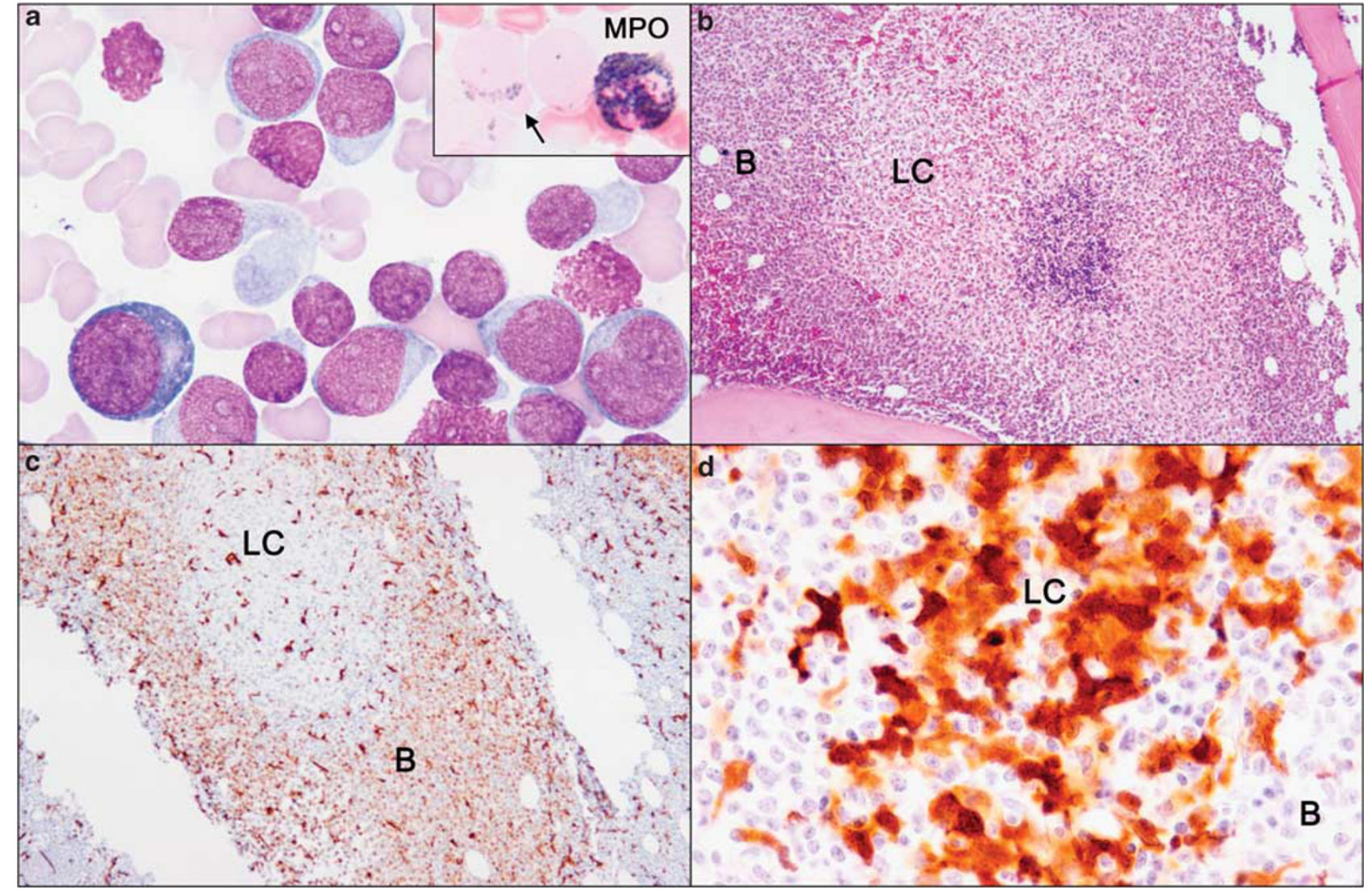

Figure 1 Patient 1 had acute myeloid leukemia, not otherwise specified (NOS) (without maturation) with T-cell antigen expression and association with Langerhans cell (LC) histiocytosis. Wright-Giemsa stain of a bone marrow aspirate (a) highlighting the leukemic blasts (B) $(100 \times)$ with the inset showing myeloperoxidase (MPO) positivity of the blasts. Targetoid lesions within the bone marrow (b) with CD68-positive monocytic blasts (c) surrounding the S100-positive LCs (d).

a limited number of cases; ${ }^{6,7}$ however, to our knowledge, a clonal relationship has not been demonstrated in LC histiocytosis associated with other acute leukemias. In this report, we describe several cases of acute leukemia of ambiguous or myeloid lineage in association with LC histiocytosis that provide evidence suggesting a common origin between the two neoplasms.

\section{Materials and methods}

Four adult patients with acute leukemia and concurrent or subsequent LC histiocytosis were identified over a period of 12 years. Original evaluation of leukemia was performed at different institutions using morphologic examination, flow cytometric immunophenotyping, immunohistochemical stains, 


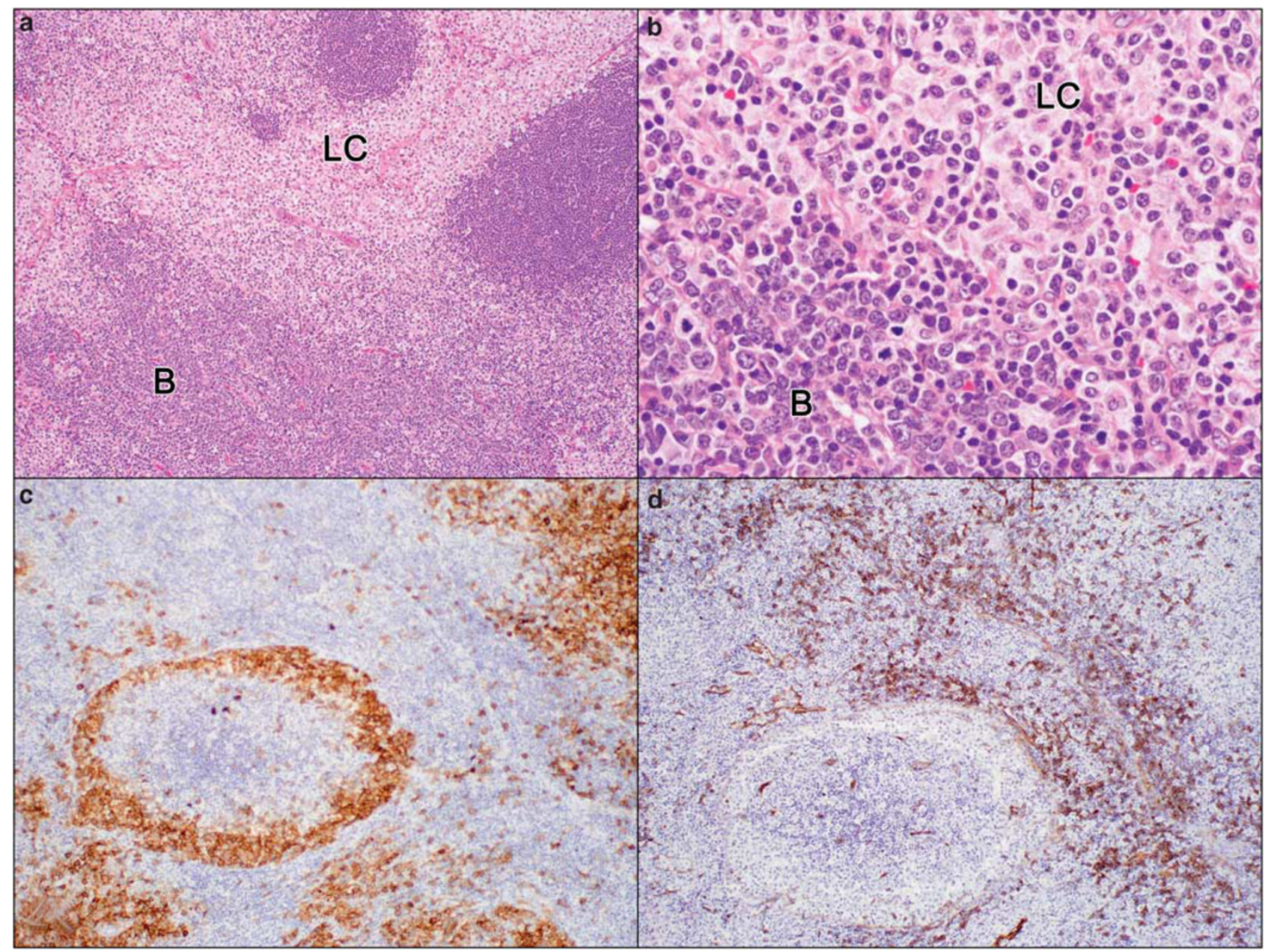

Figure 2 Biopsy of the lymph node of patient 3 shows Langerhans cell (LC) lesions mixed with blasts on hematoxylin and eosin (H\&E) at $10 \times(\mathbf{a})$ and $50 \times(\mathbf{b})$. In some areas, there are targetoid lesions in the lymph node with blasts, LCs, and lymphocytes, which is highlighted by CD1a (c) and CD34 (d). Lymphocytes are in the center with a rim of LCs and blasts with partial CD34 form the outermost area.

and cytogenetic studies. The presence of LCs was confirmed by immunohistochemistry for CD1a, S100, and langerin. FISH was performed on a lymph node of one patient who had a trisomy 21 leukemic clone.

As BRAF mutations occur in a significant percentage of LC histiocytosis, ${ }^{8}$ BRAF V600E testing was performed in three cases by real-time polymerase chain reaction (RT-PCR). DNA was extracted from formalin-fixed, paraffin-embedded tissue (two cases) or B5-fixed tissue (one case) using the DNeasy blood and tissue kit, and then diluted to a DNA concentration of $66.7 \mathrm{ng} / \mu \mathrm{l}$. The RT-PCR procedure was modified from Benlloch et $a l^{9}$ and samples were run on an ABI7900HT (Applied Biosystems, Foster City, CA, USA) with the following controls: $100 \%$ tumor, $10 \%$ tumor, negative, and no template.

\section{Results}

Four patients presented with acute leukemia and concurrent (three cases) or subsequent (one case) LC histiocytosis (see Table 1). They were all adults with a median age of 61 (range 36-80) years and three of the four patients were male. All patients either presented with or developed extramedullary leukemia in lymph nodes. Two patients (patients 1 and 4) had concurrent LC histiocytosis and leukemia in both the bone marrow and lymph node, one patient (patient 3) had concurrent LC histiocytosis and leukemia in a lymph node, and one patient (patient 2) presented with isolated leukemia in the bone marrow with subsequent, 4 months later, leukemia and LC histiocytosis in a lymph node. In all four patients, leukemic blasts and LC lesions were geographically intermingled in the involved tissue, and in patients 1 and 3, the leukemic blasts and LCs formed targetoid lesions (see Figures 1-3). In all patients, LC lesions were not isolated, but only present with leukemia.

The leukemic blasts in all cases expressed at least one T-cell marker and in two patients the leukemic blasts had an ambiguous phenotype (see Table 2). Patient 1 had acute myeloid leukemia, not otherwise specified, with the expression of three T-cell 


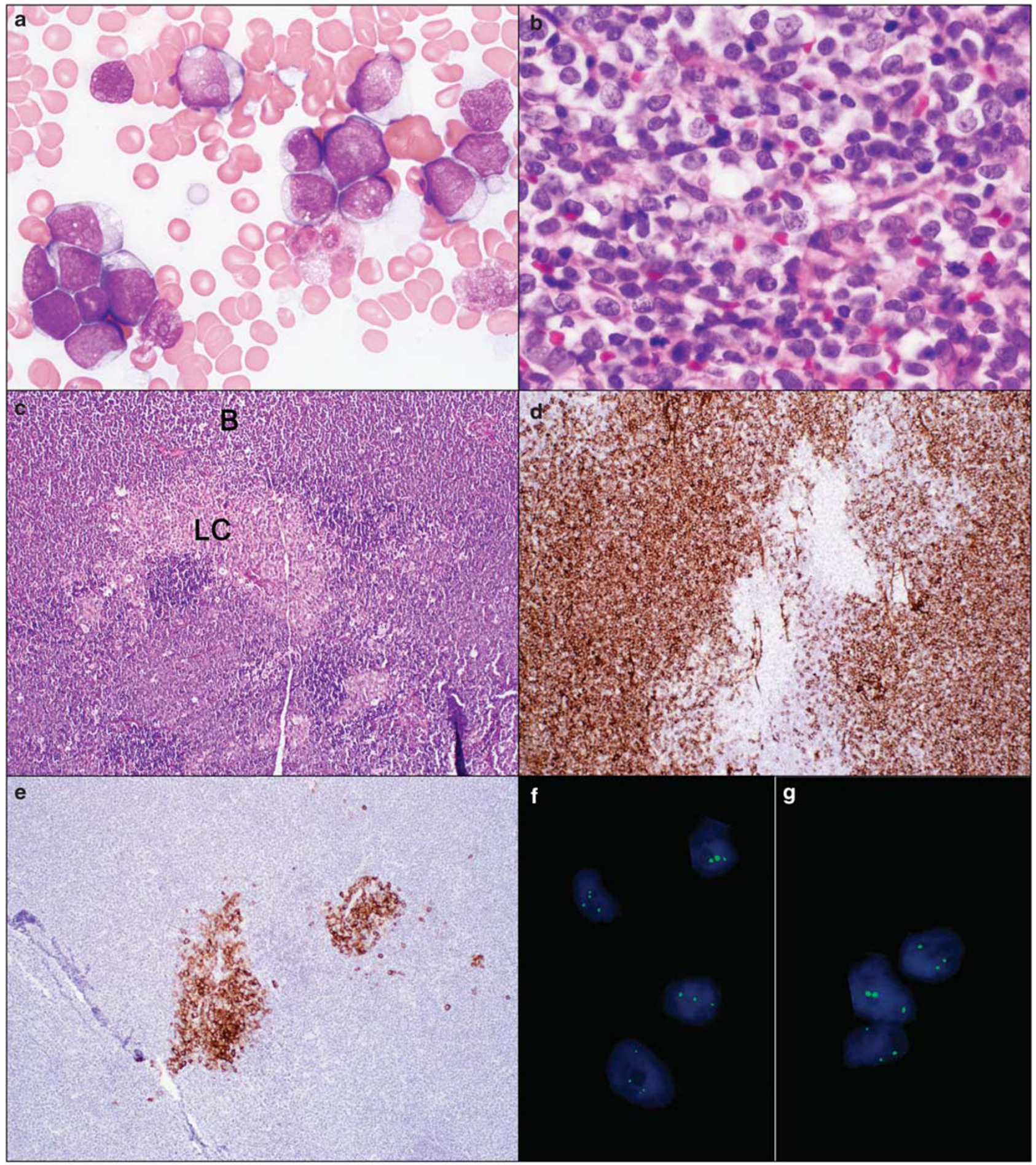

Figure 3 Patient 4 had lymph node and bone marrow involvement. The bone marrow aspirate (a) shows numerous blasts (WrightGiemsa, $100 \times$ ). The bone marrow trephine biopsy (b) shows a mixture of blasts and Langerhans cells (hematoxylin and eosin (H\&E), $50 \times$ ). The lymph node biopsy shows discrete Langerhans cell lesions $(\mathbf{c})$ that are surrounded by blasts $(\mathrm{H} \& \mathrm{E}, 10 \times)$. The blasts are CD34positive (d) and the Langerhans cells are CD1a- (e) and S100- (not shown) positive. Fluorescent in situ hybridization (FISH) in the Langerhans cell areas shows trisomy 21 (f) in approximately half the cells and FISH in the blast areas shows trisomy 21 (g) in approximately half the cells.

markers (CD2, CD5, and CD7) and TdT. Patient 2 presented with acute myeloid leukemia with myelodysplasia-related changes and developed comingled LC histiocytosis and extramedullary leukemia in a lymph node following consolidation chemotherapy. The LCs in this case (patient 2) had immunophenotypic similarities to the leukemic blasts with the expression of CD2, CD13, and CD117. 
Table 2 Immunophenotype of leukemic blasts and Langerhans cells

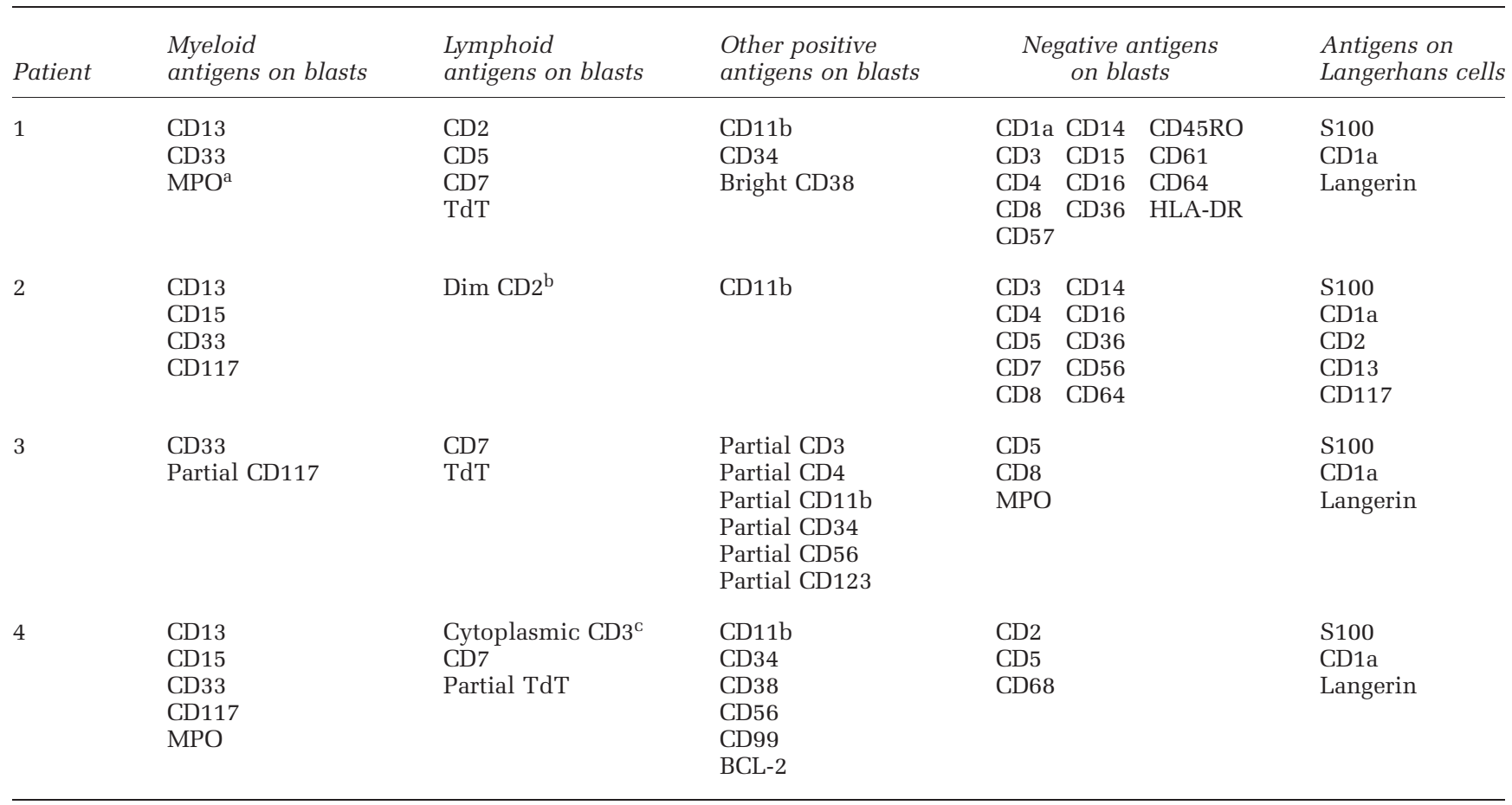

${ }^{a}$ Myeloperoxidase was present by flow cytometry and also by enzyme cytochemical staining.

${ }^{\mathrm{b}} \mathrm{CD} 2$ was expressed on the blasts in the lymph node but not the blasts in the bone marrow.

${ }^{\mathrm{C}}$ Cytoplasmic CD3 was present by immunohistochemistry and flow cytometry.

Patient 3 had undifferentiated acute leukemia with the expression of CD7 and TdT. Patient 4 had mixed phenotype T/myeloid acute leukemia by the 2008 WHO criteria ${ }^{10}$ with the expression of cytoplasmic CD3 and CD7.

One patient (patient 4) had a non-constitutional trisomy 21. FISH analysis of the involved lymph node revealed trisomy 21 in both the LCs and the blasts, supporting a clonal relationship between the two cell types. BRAF V600E analysis was successfully performed in two of the three patients tested (patients 3 and 4) and neither exhibited a mutation.

\section{Discussion}

LC proliferations associated with an underlying hematologic neoplasm may be explained by several different mechanisms including: coincidental occurrence of two distinct neoplasms, a reactive proliferation of LCs in association with another neoplasm, development of a myeloid neoplasm secondary to LC histiocytosis therapy, or a clonally related process where the LCs are derived either from a common stem cell or through transdifferentiation of the myeloid neoplasm. There is evidence that different mechanisms may exist in different patients. ${ }^{5-7}$ The biology of cases with a clonal relationship are of particular interest in understanding the lineage connection of these distinct cell types and the possible future impact on treatment of these diseases.
A clonal relationship between LC histiocytosis and T-lymphoblastic leukemia has been established in a small number of cases ${ }^{6,7}$ but has not been reported in other acute leukemias. Two of the cases reported here support the concept that concurrent or subsequent LC histiocytosis in patients with underlying hematopoietic neoplasms are, in some cases, clonally related to the underlying neoplasm. In one of the cases (patient 4), a clonal relationship of the leukemia and LC histiocytosis was established by a shared cytogenetic abnormality in the two neoplastic cell lineages by FISH studies on lymph node sections. In another case (patient 2), there is suggestive evidence of a clonal relationship with the expression of CD2, CD13, and CD117 on both the leukemic blasts and the LCs. The presence of myeloid-associated markers is unusual on LCs. ${ }^{12}$

The four patients were all adults and shared several infrequent findings that suggest a common underlying biology. Extramedullary leukemia involving lymph nodes was present in all and as a presenting feature in three. There was an intimate geographic relationship of the LC lesions and the leukemia in lymph nodes and/or bone marrow without evidence of LC histiocytosis anatomically separate from the acute leukemia. These unusual findings neither prove nor refute a clonal relationship of the leukemia and LC histiocytosis but suggest commonality among the cases.

The significance of negative BRAF V600E in the two cases is unclear as BRAF V600E can be negative 
in up to $43 \%$ of patients with LC histiocytosis. ${ }^{8}$ Young individuals with LC histiocytosis are reportedly more commonly positive for BRAF V600E. ${ }^{8}$ The two patients tested in this study were of advanced age (69 and 80 years of age).

All of the leukemias in this study were of ambiguous lineage or acute myeloid leukemia that expressed T-cell antigens. It is known that myeloid cells and dendritic cells share a common stem cell and myeloid cells can differentiate into dendritic cells. Furthermore, myeloid neoplasms including the leukemic blasts in acute myeloid leukemia can be induced to differentiate into CD1a-positive antigen-presenting dendritic cells in vitro. ${ }^{13,14}$

The new 'myeloid-based' model of hematopoiesis is one possible explanation for the unique features in our cases. This 'myeloid-based' model recognizes that myeloid potential persists even as lineage branches toward $\mathrm{T}$ and $\mathrm{B}$ cells $\mathrm{s}^{6,7,15-17}$ and proposes that $\mathrm{T}$ - and B-cell progenitors arise from common myelolymphoid progenitors through myeloid-T and myeloid-B stages. ${ }^{18,19}$ Previously published clonal relationship between LC histiocytosis and T-lymphoblastic leukemia and LC sarcoma and B-lymphoblastic leukemia further support that myeloid- $T$ and myeloid-B precursors may be the cell of origin of these types of leukemia. ${ }^{6,7,15-17}$ In 2008, Wada et al ${ }^{20}$ provided evidence that there are progenitor cells in the adult thymus that have lost the potential to generate $\mathrm{B}$ cells but retain substantial macrophage potential as well as T-cell, natural killer cell and dendritic cell potential. ${ }^{20}$ Our cases with evidence of myeloid and variable T-cell differentiation combined with a dendritic cell population (LC histiocytosis) suggests that they may have developed from this specific type of progenitor cell. The 'myeloid-based' model with myelolymphoid progenitors seems to improve on our understanding of unusual acute leukemias with mixed phenotypes as well as cases in which transdifferentiation was detected. Our cases further support this model as they show both mixed phenotypes and probable transdifferentiation.

\section{Disclosure/conflict of interest}

The authors declare no conflict of interest.

\section{References}

1 Abla O, Egeler RM, Weitzman S. Langerhans cell histiocytosis: current concepts and treatments. Cancer Treat Rev 2010;36:354-359.

2 Egeler RM, Neglia JP, Puccetti DM, et al. Association of Langerhans cell histiocytosis with malignant neoplasms. Cancer 1993;71:865-873.

3 Egeler RM, Neglia JP, Arico M, et al. Acute-leukemia in association with Langerhans cell histiocytosis. Med Pediatr Oncol 1994;23:81-85.
4 Egeler RM, Neglia JP, Arico M, et al. The relation of Langerhans cell histiocytosis to acute leukemia, lymphomas, and other solid tumors-the LCH-Malignancy Study Group of the Histiocyte Society. Hematol Oncol Clin N Am 1998;12:369-378.

5 Christie LJ, Evans AT, Bray SE, et al. Lesions resembling Langerhans cell histiocytosis in association with other lymphoproliferative disorders: a reactive or neoplastic phenomenon? Hum Pathol 2006; 37:32-39.

6 Rodig SJ, Payne EG, Degar BA, et al. Aggressive Langerhans cell histiocytosis following T-ALL: clonally related neoplasms with persistent expression of constitutively active NOTCH1. Am J Hematol 2008; 83:116-121.

7 Feldman AL, Berthold F, Arceci R, et al. Clonal relationship between precursor T-lymphoblastic leukaemia/lymphoma and Langerhans-cell histiocytosis. Lancet Oncol 2005;6:435-437.

8 Badalian-Very G, Vergilio JA, Degar BA, et al. Recurrent BRAF mutations in Langerhans cell histiocytosis. Blood 2010;116:1919-1923.

9 Benlloch S, Paya A, Alenda C, et al. Detection of BRAF V600E mutation in colorectal cancer: comparison of automatic sequencing and real-time chemistry methodology. J Mol Diagn 2006;8:540-543.

10 Swerdlow SH, Campo E, Harris NL, et al. WHO Classification of Tumours of Hematopoietic and Lymphoid Tissues, 4th edn. International Agency for Research on Cancer: Lyon, France, 2008.

11 Bene MC, Castoldi G, Knapp W, et al. Proposals for the immunological classification of acute leukemias. Leukemia 1995;9:1783-1786.

12 Caponetti GC, Miranda RN, Althof PA, et al. Immunohistochemical and molecular cytogenetic evaluation of potential targets for tyrosine kinase inhibitors in Langerhans cell histiocytosis. Hum Pathol 2012;43: 2223-2228.

13 Robinson SP, English N, Jaju R, et al. The in vitro generation of dendritic cells from blast cells in acute leukaemia. Br J Haematol 1998;103:763-771.

14 Cignetti A, Bryant E, Allione B, et al. CD34(+) acute myeloid and lymphoid leukemic blasts can be induced to differentiate into dendritic cells. Blood 1999;94: 2048-2055.

15 Kawamoto H, Ohmura K, Katsura Y. Direct evidence for the commitment of hematopoietic stem cells to T, B and myeloid lineages in murine fetal liver. Int Immunol 1997;9:1011-1019.

$16 \mathrm{Lu} \mathrm{M}$, Kawamoto H, Katsube Y, et al. The common myelolymphoid progenitor: a key intermediate stage in hemopoiesis generating $\mathrm{T}$ and B cells. J Immunol 2002; 169:3519-3525.

17 Ratei R, Hummel M, Anagnostopoulos I, et al. Common clonal origin of an acute B-lymphoblastic leukemia and a Langerhans' cell sarcoma: evidence for hematopoietic plasticity. Haematologica 2010;95: 1461-1466.

18 Katsura Y. Redefinition of lymphoid progenitors. Nat Rev Immunol 2002;2:127-132.

19 Kawamoto H. A close developmental relationship between the lymphoid and myeloid lineages. Trends Immunol 2006;27:169-175.

20 Wada H, Masuda K, Satoh R, et al. Adult T-cell progenitors retain myeloid potential. Nature 2008;452: 768-772. 